\title{
Intuitionistic Fuzzy R-Ideals of BCI-Algebras with Interval Valued Membership\& Non Membership Functions
}

\author{
C.Ragavan ${ }^{1}$, A.Solairaju ${ }^{2}$ and M.Balamurugan ${ }^{3}$ \\ ${ }^{1,3}$ Asst Prof. Department of Mathematics, Sri Vidya Mandir Arts \& Science College, Uthangarai, T.N. India. \\ ${ }^{2}$ Associate Professor of Mathematics Jamal Mohamed College, Trichy, T.N, India.
}

\begin{abstract}
The purpose of this paper is to define the notion of an interval valued Intuitionistic Fuzzy R-ideal (briefly, an i-v IF R-ideal) of a BCI - algebra. Necessary and sufficient conditions for an i-v Intuitionistic Fuzzy R-ideal are stated. Cartesian product of $i$-v Fuzzy ideals are discussed.
\end{abstract}

\section{Introduction:}

The notion of BCK-algebras was proposed by Imai and Iseki in 1996. In the same year, Iseki [6] introduced the notion of a BCI-algebra which is a generalization of a BCK-algebra. Since then numerous mathematical papers have been written investigating the algebraic properties of the $\mathrm{BCK} / \mathrm{BCI}$-algebras and their relationship with other universal structures including lattices and Boolean algebras. Fuzzy sets were initiated by Zadeh [10]. In [9], Zadeh made an extension of the concept of a Fuzzy set by an interval-valued fuzzy set. This interval-valued fuzzy set is referred to as an i-v fuzzy set. InZadeh also constructed a method of approximate inference using his i-v fuzzy sets. In Birwa's defined interval valued fuzzy subgroups of Rosenfeld`s nature, and investigated some elementary properties. The idea of "intuitionistic fuzzy set" was first published by Atanassov as a generalization of notion of fuzzy sets. After that many researchers considers the Fuzzifications of ideal and sub algebras in BCK/BCI-algebras. In this paper, using the notion of interval valued fuzzy set, we introduce the concept of an interval-valued intuitionistic fuzzy BCI-algebra of a BCI-algebra, and study some of their properties. Using an i-v level set of $\mathrm{i}-\mathrm{v}$ intuitionistic fuzzy set, we state a characterization of an intuitionistic fuzzy R-ideal of BCI-algebra. We prove that every intuitionistic fuzzy R-ideal of a BCI-algebra X can be realized as an $\mathrm{i}-\mathrm{v}$ level R-ideal of an $\mathrm{i}-\mathrm{v}$ intuitionistic fuzzyR-ideal of $\mathrm{X}$. in connection with the notion of homomorphism, we study how the images and inverse images of i-v intuitionistic fuzzy R-ideal become i-v intuitionistic fuzzy R-ideal.

\section{Preliminaries:}

Let us recall that an algebra $(X, *, 0)$ of type $(2,0)$ is called a BCI-algebra if it satisfies the following conditions:1. $\left(\left(\mathrm{x}^{*} \mathrm{y}\right) *(\mathrm{x} * \mathrm{z})\right)^{*}(\mathrm{z} * \mathrm{y})=0,2 .(\mathrm{x} *(\mathrm{x} * \mathrm{y}))^{*} \mathrm{y}=0,3 \cdot \mathrm{x} * \mathrm{x}=0,4 \cdot \mathrm{x} * \mathrm{y}=0$ and $\mathrm{y}^{*} \mathrm{x}=0$ imply $\mathrm{x}=\mathrm{y}$, for all $\mathrm{x}, \mathrm{y}, \mathrm{z} \in \mathrm{X}$. In a BCI-algebra, we can define a partial ordering" $\leq$ " by $\mathrm{x} \leq \mathrm{y}$ if and only if $\mathrm{x}^{*} \mathrm{y}=0$.in a BCI-algebra $\mathrm{X}$, the set $\mathrm{M}=\left\{\mathrm{x} \in \mathrm{X} / 0^{*} \mathrm{x}=0\right\}$ is a sub algebra and is called the BCK-part of X. A BCI-algebra $\mathrm{X}$ is called proper if $\mathrm{X}-\mathrm{M} \neq \phi$. otherwise it is improper. Moreover, in a BCI-algebra the following conditions hold:

1. $(\mathrm{x} * \mathrm{y}) * \mathrm{z}=(\mathrm{x} * \mathrm{z}) * \mathrm{y}, 2 . \mathrm{x} * 0=0,3 . \mathrm{x} \leq \mathrm{y}$ imply $\mathrm{x} * \mathrm{z} \leq \mathrm{y} * \mathrm{z}$ and $\mathrm{z} * \mathrm{y} \leq \mathrm{z} * \mathrm{x}, 4.0 *(\mathrm{x} * \mathrm{y})=(0 * \mathrm{x}) *(0 * \mathrm{y})$,

5. $0 *(\mathrm{x} * \mathrm{y})=(0 * \mathrm{x}) *(0 * \mathrm{y}), 6.0 *(0 *(\mathrm{x} * \mathrm{y}))=0 *(\mathrm{y} * \mathrm{x}), 7 .(\mathrm{x} * \mathrm{z}) *(\mathrm{y} * \mathrm{z}) \leq \mathrm{x} * \mathrm{y}$

An intuitionistic fuzzy set $A$ in a non-empty set $X$ is an object having the form $A=$ $\left\{\left\langle\mathrm{x}, \mu_{\mathrm{A}}(\mathrm{x}), v_{\mathrm{A}}(\mathrm{x})>/ \mathrm{x} \in \mathrm{X}\right\}\right.$, Where the functions $\mu_{\mathrm{A}}: \mathrm{X} \rightarrow[0,1]$ and $v_{\mathrm{A}}: \mathrm{X} \rightarrow[0,1]$ denote the degree of the membership and the degree of non membershipof each element $\mathrm{x} \epsilon \mathrm{X}$ to the set A respectively, and $0 \leq \mu_{\mathrm{A}}(\mathrm{x})$ $+v_{\mathrm{A}}(\mathrm{x}) \leq 1$ for all $\mathrm{x} \in \mathrm{X}$. Such defined objects are studied by many authors and have many interesting applications not only in the mathematics. For the sake of simplicity, we shall use the symbol $A=\left[\mu_{A}, v_{A}\right]$ for the intuitionistic fuzzy set $\mathrm{A}=\left\{\left[\mu_{\mathrm{A}}(\mathrm{x}), v_{\mathrm{A}}(\mathrm{x})\right] / \mathrm{x} \in \mathrm{X}\right\}$.

Definition 2.1:A non empty subset $I$ of $X$ is called an ideal of $X$ if it satisfies:1. $0 \epsilon I, 2 . x^{*} y \in I$ and $y \in I \Rightarrow x \in I$.

Definition 2.2: A fuzzy subset $\mu$ of a BCI-algebra $X$ is called afuzzy ideal of $X$ if it satisfies:

1. $\mu(0) \geq \mu(\mathrm{x}), 2 . \mu(\mathrm{x}) \geq \min \left\{\mu\left(\mathrm{x}^{*} \mathrm{y}\right), \mu(\mathrm{y})\right\}$, for all $\mathrm{x}, \mathrm{y} \in \mathrm{X}$.

Definition 2.3: A non empty subset $I$ of $X$ is called anR- ideal of $X$ if it satisfies:

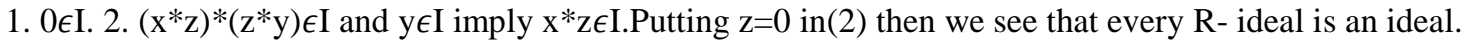

Definition 2.4: A fuzzy set $\mu$ in a BCI-algebra $X$ is called an fuzzyR- ideal of $X$ if

1. $\mu(0) \geq \mu(\mathrm{x}), 2 . \mu(\mathrm{x}) \geq \min \left\{\mu\left(\left(\mathrm{x}^{*} \mathrm{z}\right) *\left(\mathrm{z}^{*} \mathrm{y}\right)\right), \mu(\mathrm{y})\right\}$.

Definition 2.5: Let A and B be two fuzzy ideal of BCI algebra X. The fuzzy set $A \cap B$ membership function

$\mu_{A \cap B}$ is defined by $\mu_{A \cap B}(\mathrm{x})=\min \left\{\mu_{A}(\mathrm{x}), \mu_{B}(\mathrm{x})\right\}, x \in X$. 
Definition 2.6: Let $A$ and B be two fuzzy ideal of BCI algebra X. The fuzzy set $A \cup B$ with membership function $\mu_{A \cup B}$ is defined by $\mu_{A \cup B}(\mathrm{x})=\max \left\{\mu_{A}(\mathrm{x}), \mu_{B}(\mathrm{x})\right\}, \forall x \in X$.

Definition 2.7: Let $A$ and $B$ be two fuzzy ideal of BCI algebra $X$ with membership functionand respectively. A is contained in $\mathrm{B}$ if $\mu_{A}(\mathrm{x}) \leq \mu_{B}(\mathrm{x}), \forall x \in X$

Definition 2.8: Let A be a fuzzy ideal of BCI algebra X.The fuzzy set $A^{m}$ with membership function $\mu_{A^{m}}$ is defined by $\mu_{A^{m}}(\mathrm{x}) \leq\left(\mu_{A}(\mathrm{x})\right)^{m}, \forall x \in X$

Definition 2.9: An IFS $A=<X, \mu_{A}, v_{A}>$ in a BCI-algebra $X$ is called an intuitionisticfuzzy ideal of $X$ if it satisfies:(F1) $\mu_{\mathrm{A}}(0) \geq \mu_{\mathrm{A}}(\mathrm{x}) \& v_{\mathrm{A}}(0) \geq v_{\mathrm{A}}(\mathrm{x}),(\mathrm{F} 2) \mu_{\mathrm{A}}(\mathrm{x}) \geq \min \left\{\mu_{\mathrm{A}}\left(\mathrm{x}^{*} \mathrm{y}\right), \mu_{\mathrm{A}}(\mathrm{y})\right\}$,

(F3) $v_{\mathrm{A}}(\mathrm{x}) \leq \max \left\{v_{\mathrm{A}}\left(\mathrm{x}^{*} \mathrm{y}\right), v_{\mathrm{A}}(\mathrm{y})\right\}$, for all $\mathrm{x}, \mathrm{y} \in \mathrm{X}$.

Definition 2.10: An intuitionistic fuzzy set $A=<\mu_{A}, v_{A}>$ of a BCI-algebra $X$ is called an intuitionistic fuzzy Rideal if it satisfies $(\mathrm{F} 1)$ and $(\mathrm{F} 4) \mu_{\mathrm{A}}(\mathrm{x}) \geq \min \left\{\mu_{\mathrm{A}}\left(\left(\mathrm{x}^{*} \mathrm{z}\right) *\left(\mathrm{z}^{*} \mathrm{y}\right)\right), \mu_{\mathrm{A}}(\mathrm{y})\right\}$,

(F5) $v_{\mathrm{A}}(\mathrm{y} * \mathrm{x}) \leq \max \left\{v_{\mathrm{A}}\left((\mathrm{x} * \mathrm{z}) *\left(\mathrm{z}^{*} \mathrm{y}\right)\right), \mathrm{v}_{\mathrm{A}}(\mathrm{y})\right\}$, for all $\mathrm{x}, \mathrm{y}, \mathrm{z} \in \mathrm{X}$.

An interval-valued intuitionistic fuzzy set $A$ defined on $X$ is given by $A=\left\{\left(\mathrm{x},\left[\mu_{A}^{L}(\mathrm{x}) \mu_{A}^{U}(\mathrm{x})\right],\left[v_{A}^{L}(\mathrm{x}) v_{A}^{U}(\mathrm{x})\right]\right)\right\}, \forall x \in X$ where $\mu_{A}^{L}, \mu_{A}^{U}$ are two membership functions and $v_{A}^{L}, v_{A}^{U}$ aretwo nonmembership functions $\mathrm{X}$ such that $\mu_{A}^{L} \leq \mu_{A}^{U} \& v_{A}^{L} \geq v_{A}^{U}, \forall \mathrm{x} \in \mathrm{X}$. Let $\bar{\mu}_{\mathrm{A}}(\mathrm{x})=\left[\mu_{A}^{L}, \mu_{A}^{U}\right] \& \overline{v_{A}}(\mathrm{x})=\left[v_{A}^{L}, v_{A}^{U}\right], \forall \mathrm{x} \in \mathrm{X}$ and let $\mathrm{D}[0,1]$ denote the family of all closed subintervals of $[0,1]$.If $\mu_{A}^{L}(\mathrm{x})=\mu_{A}^{U}(\mathrm{x})=\mathrm{c}, 0 \leq \mathrm{c} \leq 1$ and if $v_{A}^{L}(\mathrm{x})=\mathrm{v}_{A}^{U}(\mathrm{x})=\mathrm{k}$, $0 \leq \mathrm{k} \leq 1$, then we have $\bar{\mu}_{\mathrm{A}}(\mathrm{x})=[\mathrm{c}, \mathrm{c}] \& \bar{v}_{\mathrm{A}}(\mathrm{x})=[\mathrm{k}, \mathrm{k}]$ which we also assume, for the sake of convenience, to belong to $\mathrm{D}[0,1]$. thus $\bar{\mu}_{\mathrm{A}}(\mathrm{x}) \& \bar{v}_{\mathrm{A}}(\mathrm{x}) \epsilon[0,1], \forall \mathrm{x} \in \mathrm{X}$, and therefore the $\mathrm{i}-\mathrm{v}$ IFS a is given by $\mathrm{A}=\left[\left(\mathrm{x}, \bar{\mu}_{\mathrm{A}}(\mathrm{x}), \bar{v}_{\mathrm{A}}(\mathrm{x})\right)\right\}, \forall \mathrm{x} \in \mathrm{X}$, where $\bar{\mu}_{\mathrm{A}}(\mathrm{x}): \mathrm{X} \rightarrow \mathrm{D}[0,1]$. Now let us define what is known as refined minimum, refined maximum of two elements in $\mathrm{D}[0,1]$.we also define the symbols" $\leq ", \geq "$ and "=" in the case of two elements in $\mathrm{D}[0,1]$. Consider two elements $\quad D_{1}:\left[a_{1}, b_{1}\right]$ and $\quad D_{2}:\left[a_{2}, b_{2}\right] \epsilon D[0,1]$ Then $\quad \operatorname{rmin}\left(D_{1}, D_{2}\right)=\left[\min \left\{a_{1}, a_{2}\right\}, \min \left\{b_{1}, b_{2}\right\}\right]$, $\operatorname{rmax}\left(\mathrm{D}_{1}, \mathrm{D} 2\right)=\left[\max \left\{\mathrm{a}_{1}, \mathrm{a}_{2}\right\}, \max \left\{\mathrm{b}_{1}, \mathrm{~b}_{2}\right\}\right]$

$\mathrm{D}_{1} \geq \mathrm{D}_{2} \Leftrightarrow \mathrm{a}_{1} \geq \mathrm{a}_{2}, \mathrm{~b}_{1} \geq \mathrm{b}_{2} ; \mathrm{D}_{1} \leq \mathrm{D}_{2} \Leftrightarrow \mathrm{a}_{1} \leq \mathrm{a}_{2}, \mathrm{~b}_{1} \leq \mathrm{b}_{2}$ and $\mathrm{D}_{1}=\mathrm{D}_{2}$.

\section{Interval-valued Intuitionistic fuzzy R-ideals of BCI-algebras}

Definition 3.1:An interval-valued intuitionistic fuzzy set $A$ in $B C I$-algebra $X$ is called an interval-valued intuitionistic fuzzy a-ideal of $X$ if it satisfies $\left(\mathrm{FI}_{1}\right) \bar{\mu}_{\mathrm{A}}(0) \geq \bar{\mu}_{\mathrm{A}}(\mathrm{x}), \bar{v}_{\mathrm{A}}(0) \leq \bar{v}_{\mathrm{A}}(\mathrm{x}),\left(\mathrm{FI}_{2}\right) \bar{\mu}_{\mathrm{A}}(\mathrm{x})$

$\geq \mathrm{r} \min \left\{\bar{\mu}_{\mathrm{A}}\left((\mathrm{x} * \mathrm{z})^{*}\left(\mathrm{z}^{*} \mathrm{y}\right)\right), \bar{\mu}_{\mathrm{A}}(\mathrm{y})\right\},\left(\mathrm{FI}_{3}\right) \bar{v}_{\mathrm{A}}(\mathrm{x}) \leq \mathrm{r} \max \left\{\bar{v}_{\mathrm{A}}\left(\left(\mathrm{x}^{*} \mathrm{z}\right)^{*}\left(\mathrm{z}^{*} \mathrm{y}\right)\right), \overline{\mathrm{v}}_{\mathrm{A}}(\mathrm{y})\right\}$.

Theorem 3.2Let $A$ be an $i-v$ intuitionistic fuzzyR-ideal of $X$. if there exists a sequence $\left\{x_{n}\right\}$ in $X$ such that

$\lim _{n \rightarrow \infty} \bar{\mu}_{A}\left(x_{n}\right)=[1,1], \lim _{n \rightarrow \infty} \bar{\nu}_{A}\left(x_{n}\right)=[0,0]$ then $\bar{\mu}_{\mathrm{A}}(0)=[1,1]$ and $\bar{v}_{\mathrm{A}}(0)=[0,0]$.

Proof:Since $\bar{\mu}_{A}(0) \geq \bar{\mu}_{A}(x)$ and $\bar{v}_{A}(0) \leq \bar{v}_{A}(x)$ for all $x \in X$, we have $\bar{\mu}_{A}(0) \geq \bar{\mu}_{A}\left(x_{n}\right)$ and $\bar{v}_{A}(0) \leq \bar{v}_{A}\left(x_{n}\right)$, for every positive integer $\mathrm{n}$. note that $\left[\mu_{A}^{L}, \mu_{A}^{U}\right] \geq \bar{\mu}_{A}(0) \cdot[1,1] \geq \bar{\mu}_{\mathrm{A}}(\mathrm{x}) \geq \bar{\mu}_{\mathrm{A}}(0) \geq \lim _{n \rightarrow \infty} \bar{\mu}_{A}\left(x_{n}\right)=[1,1] .\left[\lambda_{A}^{L}, \lambda_{A}^{U}\right] \leq \bar{\lambda}_{A}(0)$

$.[0,0] \leq \bar{v}_{\mathrm{A}}(\mathrm{x}) \leq \bar{v}_{\mathrm{A}}(0) \leq \lim _{n \rightarrow \infty} \bar{v}_{A}\left(x_{n}\right)=[0,0]$. Hence $\bar{\mu}_{\mathrm{A}}(0)=[1,1]$ and $\bar{v}_{\mathrm{A}}(0)=[0,0]$.

Lemma3.3:An i-v intuitionistic fuzzy set $\mathrm{A}=\left[\left\langle\mu_{A}^{L}, \mu_{A}^{U}\right\rangle,\left\langle v_{A}^{L}, v_{A}^{U}\right\rangle\right]$ in $\mathrm{X}$ is an i-v intuitionistic fuzzy R-ideal of $\mathrm{X}$ if and only if $\left\langle\mu_{A}^{L}, \mu_{A}^{U}\right\rangle$ and $\left\langle v_{A}^{L}, v_{A}^{U}\right\rangle$ are intuitionistic fuzzy ideals of $X$.

Proof:Since $\mu_{A}^{L}(0) \geq \mu_{A}^{L}(\mathrm{x}) ; \mu_{A}^{U}(0) \geq \mu_{A}^{U}(\mathrm{x}) ; v_{A}^{L}(0) \leq v_{A}^{L}(x)$ and $v_{A}^{U}(0) \leq v_{A}^{U}(x)$, therefore $\bar{\mu}_{\mathrm{A}}(0) \geq \bar{\mu}_{\mathrm{A}}(\mathrm{x})$,

$\overline{\mathrm{v}}_{\mathrm{A}}(0) \leq \overline{\mathrm{v}}_{\mathrm{A}}(\mathrm{x})$. Suppose that $\left\langle\mu_{A}^{L}, \mu_{A}^{U}\right\rangle$ and $\left\langle v_{A}^{L}, v_{A}^{U}\right\rangle$ are intuitionistic fuzzy ideal of $\mathrm{X}$. let $\mathrm{x}, \mathrm{y} \in \mathrm{X}$, then

$\bar{\mu}_{\mathrm{A}}(\mathrm{x})=\left[\mu_{A}^{L}(\mathrm{x}), \mu_{A}^{U}(\mathrm{x})\right] \geq\left[\min \left\{\mu_{A}^{L}\left(\mathrm{x}^{*} \mathrm{y}\right), \mu_{A}^{L}(\mathrm{y})\right\}, \min \left\{\mu_{A}^{U}(\mathrm{x} * \mathrm{y}), \mu_{A}^{U}(\mathrm{y})\right\}\right]$

$=\mathrm{r} \min \left\{\left[\mu_{A}^{L}\left(\mathrm{x}^{*} \mathrm{y}\right), \mu_{A}^{U}\left(\mathrm{x}^{*} \mathrm{y}\right)\right],\left[\mu_{A}^{L}(\mathrm{y}), \mu_{A}^{U}(\mathrm{y})\right]\right\}$

$=\mathrm{r} \min \left\{\bar{\mu}_{\mathrm{A}}\left(\mathrm{x}^{*} \mathrm{y}\right), \bar{\mu}_{\mathrm{A}}(\mathrm{y})\right\}$ and

$\overline{\mathrm{v}}_{\mathrm{A}}(\mathrm{x})=\left[\mathrm{v}_{A}^{L}(\mathrm{x}), \mathrm{v}_{A}^{U}(\mathrm{x})\right] \leq\left[\max \left\{\mathrm{v}_{A}^{L}\left(\mathrm{x}^{*} \mathrm{y}\right), v_{A}^{L}(\mathrm{y})\right\}, \max \left\{\mathrm{v}_{A}^{U}\left(\mathrm{x}^{*} \mathrm{y}\right), \mathrm{v}_{A}^{U}(\mathrm{y})\right\}\right]$

$=\mathrm{r} \max \left\{\left[v_{A}^{L}\left(\mathrm{x}^{*} \mathrm{y}\right), \mathrm{v}\left(\mathrm{x}^{*} \mathrm{y}\right)\right],\left[v_{A}^{L}(\mathrm{y}), \mathrm{v}(\mathrm{y})\right]\right\}$

Conversely,

$=\mathrm{r} \max \left\{\bar{v}_{\mathrm{A}}\left(\mathrm{x}^{*} \mathrm{y}\right), \overline{\mathrm{v}}_{\mathrm{A}}(\mathrm{y})\right\}$. Hence $\mathrm{A}$ is an $\mathrm{i}-\mathrm{v}$ intuitionistic fuzzy ideal of $\mathrm{X}$.

Assume that $\mathrm{A}$ is an $\mathrm{i}-\mathrm{v}$ intuitionistic fuzzy ideal of $\mathrm{X}$. for any $\mathrm{x}, \mathrm{y} \in \mathrm{X}$, we have

$\left[\mu_{A}^{L}(\mathrm{x}), \mu_{A}^{U}(\mathrm{x})\right]=\bar{\mu} \mathrm{A}(\mathrm{x}) \geq \mathrm{r} \min \left\{\left[\bar{\mu}_{\mathrm{A}}\left(\mathrm{x}^{*} \mathrm{y}\right), \bar{\mu}_{\mathrm{A}}(\mathrm{y})\right]\right\}$

$=\mathrm{r} \min \left\{\left[\mu_{A}^{L}\left(\mathrm{x}^{*} \mathrm{y}\right), \mu_{A}^{U}\left(\mathrm{x}^{*} \mathrm{y}\right)\right],\left[\mu_{A}^{L}(\mathrm{y}), \mu_{A}^{U}(\mathrm{y})\right]\right\}$

$=\left[\min \left\{\mu_{A}^{L}\left(\mathrm{x}^{*} \mathrm{y}\right), \mu_{A}^{L}(\mathrm{y})\right\}, \min \left\{\mu_{A}^{U}\left(\mathrm{x}^{*} \mathrm{y}\right), \mu_{A}^{U}(\mathrm{y})\right\}\right]$

And $\left[v_{A}^{L}(\mathrm{x}), \mathrm{v}_{A}^{U}(\mathrm{x})\right]=\overline{\mathrm{v}}_{\mathrm{A}}(\mathrm{x}) \leq \mathrm{r} \max \left\{\overline{\mathrm{v}}_{\mathrm{A}}\left(\mathrm{x}^{*} \mathrm{y}\right), \overline{\mathrm{v}}_{\mathrm{A}}(\mathrm{y})\right\}$

$=\mathrm{r} \max \left\{\left[v_{A}^{L}\left(\mathrm{x}^{*} \mathrm{y}\right), \mathrm{v}_{A}^{U}\left(\mathrm{x}^{*} \mathrm{y}\right)\right],\left[v_{A}^{L}(\mathrm{y}), v_{A}^{U}(\mathrm{y})\right]\right\}$

$=\left[\max \left\{v_{A}^{L}\left(\mathrm{x}^{*} \mathrm{y}\right), v_{A}^{L}(\mathrm{y})\right\}, \min \left\{v_{A}^{U}\left(\mathrm{x}^{*} \mathrm{y}\right), v_{A}^{U}(\mathrm{y})\right\}\right]$ 
It follows that $\mu_{A}^{L}(\mathrm{x}) \geq \min \left\{\mu_{A}^{L}(\mathrm{x} * \mathrm{y}), \mu_{A}^{L}(\mathrm{y})\right\}, v_{A}^{L}(\mathrm{x}) \leq \max \left\{v_{A}^{L}\left(\mathrm{x}^{*} \mathrm{y}\right), v_{A}^{L}(\mathrm{y})\right\}$

$\operatorname{And} \mu_{A}^{U}(\mathrm{x}) \geq \min \left\{\mu_{A}^{U}(\mathrm{x} * \mathrm{y}), \mu_{A}^{U}(\mathrm{y})\right\}, v_{A}^{U}(\mathrm{x}) \leq \max \left\{v_{A}^{U}\left(\mathrm{x}^{*} \mathrm{y}\right), v_{A}^{U}(\mathrm{y})\right\}$

Hence $\left\langle\mu_{A}^{L}, \mu_{A}^{U}\right\rangle$ and $\left\langle v_{A}^{L}, v_{A}^{U}\right\rangle$ are intuitionistic fuzzy ideals of $\mathrm{X}$.

Theorem 3.4.Every i-v intuitionistic fuzzy R-ideal of a BCI-algebraX is an i-v intuitionistic fuzzy ideal.

Proof:Let $\mathrm{A}=\left[\left\langle\mu_{A}^{L}, \mu_{A}^{U}\right\rangle,\left\langle v_{A}^{L}, v_{A}^{U}\right\rangle\right]$ be an i-v intuitionistic fuzzy R-ideal of $\mathrm{X}$, where $\left\langle\mu_{A}^{L}, \mu_{A}^{U}\right\rangle$ and $\left\langle v_{A}^{L}, v_{A}^{U}\right\rangle$ are intuitionistic fuzzy R-ideal of X. thus $\left\langle\mu_{A}^{L}, \mu_{A}^{U}\right\rangle$ and $\left\langle v_{A}^{L}, v_{A}^{U}\right\rangle$ are intuitionistic fuzzy R-ideals of X. hence by lemma 3.3, A is i-v intuitionistic fuzzy ideal of $X$.

Definition 3.5:An i-v intuitionistic fussy set $\mathrm{A}$ in $\mathrm{X}$ is called an interval-valued intuitionistic fuzzy BCI-sub algebraof Xif $\bar{\mu}_{\mathrm{A}}\left(\mathrm{x}^{*} \mathrm{y}\right) \geq \mathrm{r} \min \left\{\bar{\mu}_{\mathrm{A}}(\mathrm{x}), \bar{\mu}_{\mathrm{A}}(\mathrm{y})\right\}$ and $\bar{v}_{\mathrm{A}}(\mathrm{x} * \mathrm{y}) \leq\left\{\bar{v}_{\mathrm{A}}(\mathrm{x}), \overline{\mathrm{v}}_{\mathrm{B}}(\mathrm{y})\right\}$, for all $\mathrm{x}, \mathrm{y} \in \mathrm{X}$.

Theorem 3.6: Every i-v intuitionisticfuzzy R-ideal of a BCI-algebra $X$ is i-v intuitionistic fuzzy sub algebra of $\mathrm{X}$.

Proof:Let $\mathrm{A}=\left[\left\langle\mu_{A}^{L}, \mu_{A}^{U}\right\rangle,\left\langle v_{A}^{L}, v_{A}^{U}\right\rangle\right]$ be an i-v intuitionistic fuzzy R-ideal of X, where $\left\langle\mu_{A}^{L}, \mu_{A}^{U}\right\rangle$, and $\left\langle v_{A}^{L}, v_{A}^{U}\right\rangle$ are intuitionistic fuzzy R-ideal of BCI-algebra X. thus $\left\langle\mu_{A}^{L}, \mu_{A}^{U}\right\rangle$, and $\left\langle v_{A}^{L}, v_{A}^{U}\right\rangle$ are intuitionistic fuzzy subalgebra of X. Hence, $\mathrm{A}$ is $\mathrm{i}-\mathrm{v}$ intuitionistic fuzzysub algebra of $\mathrm{X}$.

\section{Cartesian product of i-v intuitionistic fuzzy R-ideals}

Definition 4.1An intuitionistic fuzzy relation A on any set a is a intuitionistic fuzzy subset A with a membership function $\Omega_{\mathrm{A}}: \mathrm{X} \times \mathrm{X} \rightarrow[0,1]$ and non membership function $\Psi_{\mathrm{A}}: \mathrm{X} \times \mathrm{X} \rightarrow[0,1]$.

Lemma 4.2Let $\bar{\mu}_{\mathrm{A}}$ and $\bar{\mu}_{\mathrm{B}}$ be two membership functions and $\bar{v}_{\mathrm{A}}$ and $\bar{v}_{\mathrm{B}}$ be two non membership functions of each $\mathrm{x} \in \mathrm{X}$ to the $\mathrm{i}-\mathrm{v}$ subsets $\mathrm{A}$ and $\mathrm{B}$, respectively. Then $\mu_{\mathrm{A}} \times \mu_{\mathrm{B}}$ is membership function and $v_{\mathrm{A}} \times v_{\mathrm{B}}$ is non membership function of each element $(\mathrm{x}, \mathrm{y}) \epsilon \mathrm{X} \times \mathrm{X}$ to the set $\mathrm{A} \times \mathrm{B}$ and defined by $\left(\bar{\mu}_{\mathrm{A}} \times \bar{\mu}_{\mathrm{B}}\right)(\mathrm{x}, \mathrm{y})=\mathrm{r} \min \left\{\bar{\mu}_{\mathrm{A}}(\mathrm{x})\right.$, $\left.\bar{\mu}_{\mathrm{B}}(\mathrm{y})\right\}$ and

$\left(\bar{v}_{\mathrm{A}} \times \overline{\mathrm{v}}_{\mathrm{B}}\right)(\mathrm{x}, \mathrm{y})=\mathrm{r} \max \left\{\overline{\mathrm{v}}_{\mathrm{A}}(\mathrm{x}), \overline{\mathrm{v}}_{\mathrm{B}}(\mathrm{y})\right\}$.

Definition 4.3Let $\mathrm{A}=\left[\left\langle\mu_{A}^{L}, \mu_{A}^{U}\right\rangle,\left\langle v_{A}^{L}, v_{A}^{U}\right\rangle\right]$ and $\mathrm{B}=\left[\left\langle\mu_{B}^{L}, \mu_{B}^{U}\right\rangle,\left\langle v_{\square}, v_{\square}\right\rangle\right]$ be two i-v intuitionistic fuzzy subsets in a set $\mathrm{X}$. the Cartesian product of $\mathrm{A} \times \mathrm{B}$ is defined by $\mathrm{A} \times \mathrm{B}=\left\{\left((\mathrm{x}, \mathrm{y}), \bar{\mu}_{\mathrm{A}} \times \bar{\mu}_{\mathrm{B}}, \bar{v}_{\mathrm{A}} \times \bar{v}_{\mathrm{B}}\right) ; \forall \mathrm{x}, \mathrm{y} \square \mathrm{X} \times \mathrm{X}\right\}$ Where

$\mathrm{A} \times \mathrm{B}: \mathrm{X} \times \mathrm{X} \rightarrow \mathrm{D}[0,1]$.

Theorem 4.4.Let $\mathrm{A}=\left[\left\langle\mu_{\square}^{\square}, \mu_{\square}^{\square}\right\rangle,\left\langle v_{\square}, v_{\square}\right\rangle\right]$ and $\mathrm{B}=\left[\left\langle\mu_{\square}^{\square}, \mu_{\square}^{\square}\right\rangle,\left\langle v_{\square}, v_{\square}\right\rangle\right]$ be two i-v intuitionistic fuzzy subsets in a set $\mathrm{X}$,then $\mathrm{A} \times \mathrm{B}$ is an $\mathrm{i}-\mathrm{v}$ intuitionistic fuzzy a-ideal of $\mathrm{X} \times \mathrm{X}$.

Proof: $\operatorname{Let}(\mathrm{x}, \mathrm{y}) \square \mathrm{X} \times \mathrm{X}$, then by definition

$$
\begin{aligned}
& \left(\bar{\mu}_{\mathrm{A}} \times \bar{\mu}_{\mathrm{B}}\right)(0,0)=\mathrm{r} \min \left\{\bar{\mu}_{\mathrm{A}}(0), \bar{\mu}_{\mathrm{B}}(\mathrm{o})=\mathrm{r} \min \left\{\left[\mu_{\square}^{\square}(0), \mu_{\square} \square(0)\right],\left[\mu_{\square}^{\square}(0), \mu \square(0)\right]\right\}\right. \\
& =\left[\min \left\{\mu_{\square}^{\square}(0), \mu \square(0)\right\}, \min \left\{\mu_{\square}^{\square}(0), \mu_{\square}^{\square}(0)\right\}\right] \\
& \geq\left[\min \left\{\mu_{\square}^{\square}(\mathrm{x}), \mu_{\square}^{\square}(\mathrm{y})\right\}, \min \left\{\mu_{\square}^{\square}(\mathrm{x}), \mu_{\square}^{\square}(\mathrm{y})\right\}\right] \\
& =\mathrm{r} \min \left\{\left[\mu_{\square}^{\square}(\mathrm{x}), \mu_{\square}^{\square}(\mathrm{x})\right],\left[\mu_{\square}^{\square}(\mathrm{y}), \mu_{\square}^{\square}(\mathrm{y})\right]\right\} \\
& =\mathrm{r} \min \left\{\bar{\mu}_{\mathrm{A}}(\mathrm{x}), \bar{\mu}_{\mathrm{B}}(\mathrm{y})\right\}=\left(\bar{\mu}_{\mathrm{A}} \times \bar{\mu}_{\mathrm{B}}\right)(\mathrm{x}, \mathrm{y}) \\
& =\mathrm{r} \max \left\{\left[v_{\square}(0), v_{\square}^{\square}(0)\right],\left[v_{\square} \square(0), v \square(0)\right]\right\} \\
& =\left[\max \{v \square(0), v \square(0)\}, \max \left\{v_{\square}^{\square}(0), v \square(0)\right\}\right] \\
& \leq\left[\max \left\{v_{\square}^{\square}(\mathrm{x}), v_{\square}(\mathrm{y})\right\}, \max \left\{v_{\square}(\mathrm{x}), v_{\square}(\mathrm{y})\right\}\right] \\
& =\mathrm{r} \max \left\{\left[v_{\square}^{\square}(\mathrm{x}), v_{\square}^{\square}(\mathrm{x})\right],\left[v_{\square}^{\square}(\mathrm{y}), v_{\square}^{\square}(\mathrm{y})\right]\right\} \\
& =\mathrm{r} \max \left\{\bar{v}_{\mathrm{A}}(\mathrm{x}), \bar{v}_{\mathrm{B}}(\mathrm{y})=\left(\overline{\mathrm{v}}_{\mathrm{A}} \times \overline{\mathrm{v}}_{\mathrm{B}}\right)(\mathrm{x}, \mathrm{y})\right.
\end{aligned}
$$

Therefore $\left(\mathrm{FI}_{2}\right)$ holds.Now, for all $\mathrm{x}, \mathrm{y}, \mathrm{z} \square \mathrm{X}$, we have

$\left(\bar{\mu}_{\mathrm{A}} \times \bar{\mu}_{\mathrm{B}}\right)\left(\left(\mathrm{x}, \mathrm{x}^{\prime}\right)\right)=\mathrm{r} \min \left\{\mu_{\mathrm{A}}(\mathrm{x}), \mu \mathrm{B}\left(\mathrm{x}^{\prime}\right)\right\}$

$$
\begin{aligned}
& \geq r \min \left\{r \min \left\{\bar{\mu}_{A}\left(\left(x^{*} z\right)^{*}\left(\mathrm{z}^{*} y\right)\right), \bar{\mu}_{A}(\mathrm{y})\right\}, r \min \left\{\bar{\mu}_{A}\left(\left(x^{1} * z^{1}\right) *\left(\mathrm{z}^{1} * y^{1}\right)\right), \bar{\mu}_{A}\left(\mathrm{y}^{1}\right)\right\}\right\} \\
& =r \min \left\{\left\{\min \left\{\mu_{A}^{L}\left(\left(x^{*} z\right)^{*}\left(\mathrm{z}^{*} y\right)\right), \mu_{A}^{L}(\mathrm{y})\right\}, \min \left\{\mu_{A}^{U}\left(\left(x^{*} z\right)^{*}\left(\mathrm{z}^{*} y\right)\right), \mu_{A}^{U}(\mathrm{y})\right\}\right\},\right. \\
& \left\{\min \left\{\mu_{B}^{L}\left(\left(x^{1} * z^{1}\right) *\left(z^{1} * y^{1}\right)\right), \mu_{B}^{L}\left(y^{1}\right)\right\}, \min \left\{\mu_{B}^{U}\left(\left(x^{1} * z^{1}\right) *\left(z^{1 *} y^{1}\right)\right), \mu^{U}{ }_{B}\left(y^{1}\right)\right\}\right\} \\
& =\left\{\min \left\{\min \left\{\mu_{A}^{L}\left(\left(x^{*} z\right) *\left(\mathrm{z}^{*} y\right)\right), \mu_{B}^{L}\left(\left(x^{1} * z^{1}\right) *\left(z^{1} * y^{1}\right)\right)\right\}, \min \left\{\mu_{A}^{L}(\mathrm{y}), \mu_{B}^{L}\left(\mathrm{y}^{1}\right)\right\}\right\},\right. \\
& \left.\min \left\{\min \left\{\mu_{A}^{U}\left((x * z) *\left(\mathrm{z}^{*} y\right)\right), \mu_{B}^{U}\left(\left(x^{1} * z^{1}\right) *\left(z^{1 *} y^{1}\right)\right)\right\}, \min \left\{\mu_{A}^{U}(\mathrm{y}), \mu_{B}^{U}\left(\mathrm{y}^{1}\right)\right\}\right\}\right\} \\
& =\mathrm{r} \min \left\{\left(\bar{\mu}_{\mathrm{A}} \times \bar{\mu}_{\mathrm{B}}\right)\left(\left(\left(\mathrm{x}^{*} \mathrm{z}\right)^{*}\left(\mathrm{z}^{*} \mathrm{y}\right)\right),\left(\left(x^{1} * z^{1}\right) *\left(z^{1} * y^{1}\right)\right)\right),\left(\bar{\mu}_{\mathrm{A}} \times \bar{\mu}_{\mathrm{B}}\right)\left(\mathrm{y}, \mathrm{y}^{\prime}\right)\right\}
\end{aligned}
$$

Also, $\left(\bar{v}_{\mathrm{A}} \times \bar{v}_{\mathrm{B}}\right)\left(\left(\mathrm{x}, \mathrm{x}^{\prime}\right)\right)=\mathrm{r} \max \left\{\mathrm{v}_{\mathrm{A}}(\mathrm{x}), \mathrm{v}_{\mathrm{B}}\left(\mathrm{x}^{\prime}\right)\right\}$

$$
\leq r \max \left\{r \max \left\{\bar{v}_{A}\left((x * z) *\left(\mathrm{z}^{*} y\right)\right), \bar{v}_{A}(\mathrm{y})\right\}, r \max \left\{\bar{v}_{A}\left(\left(x^{1} * z^{1}\right) *\left(z^{1} * y^{1}\right)\right), \bar{v}_{A}\left(\mathrm{y}^{1}\right)\right\}\right\}
$$

$=r \max \left\{\left\{\max \left\{v_{A}^{L}\left(\left(x^{*} z\right) *\left(\mathrm{z}^{*} y\right)\right), v_{A}^{L}(\mathrm{y})\right\}, \max \left\{v_{A}^{U}\left(\left(x^{*} z\right) *\left(\mathrm{z}^{*} y\right)\right), v_{A}^{U}(\mathrm{y})\right\}\right\}\right.$,

$\left\{\max \left\{v_{B}^{L}\left(\left(x^{1} * z^{1}\right) *\left(z^{1} * y^{1}\right)\right), v_{B}^{L}\left(y^{1}\right)\right\}, \max \left\{v_{B}^{U}\left(\left(x^{1} * z^{1}\right) *\left(z^{1} * y^{1}\right)\right), v_{B}^{U}\left(y^{1}\right)\right\}\right\}$ 


$$
\begin{gathered}
=\left\{\max \left\{\max \left\{v_{A}^{L}\left(\left(x^{*} z\right) *\left(\mathrm{z}^{*} y\right)\right), v_{B}^{L}\left(\left(x^{1} * z^{1}\right) *\left(\mathrm{z}^{1} y^{1}\right)\right)\right\}, \max \left\{v_{A}^{L}(\mathrm{y}), v_{B}^{L}\left(\mathrm{y}^{1}\right)\right\}\right\},\right. \\
\left.\max \left\{\max \left\{v_{A}^{U}\left(\left(x^{*} z\right) *\left(z^{*} y\right)\right), v_{B}^{U}\left(\left(x^{1} z^{1}\right) *\left(z^{1} y^{1}\right)\right)\right\}, \max \left\{v_{A}^{U}(\mathrm{y}), v_{B}^{U}\left(\mathrm{y}^{1}\right)\right\}\right\}\right\} \\
=r \max \left\{\left(\bar{v}_{A} \times \bar{v}_{B}\right)\left(\left(\left(\mathrm{x}^{*} \mathrm{z}\right)^{*}\left(\mathrm{z}^{*} \mathrm{y}\right)\right),\left(\left(\mathrm{x}^{1 *} \mathrm{z}^{1}\right) *\left(\mathrm{z}^{1 *} \mathrm{y}^{1}\right)\right),\left(\bar{v}_{A} \times \bar{v}_{B}\right)\left(\mathrm{y}, y^{1}\right)\right\}\right.
\end{gathered}
$$

Hence $A \times B$ is an i-v intuitionistic fuzzy R-ideal of $X \times X$

Definition 4.5:Let $\bar{\mu}_{\mathrm{B}}, \bar{v}_{\mathrm{B}}$ respectively, be an i-v membership and non membership function of each element $\mathrm{X} \square \mathrm{X}$ to the set $\mathrm{B}$. then strongest $\mathrm{i}-\mathrm{v}$ intuitionistic fuzzy set relationon $\mathrm{X}$, that is a membership function relation $\bar{\mu}_{\mathrm{A}}$ on $\bar{\mu}_{\mathrm{B}}$ and non membership function relation $\bar{v}_{\mathrm{A}}$ onv $\overline{\mathrm{B}}_{\mathrm{B}}$ and $\mu_{A_{B}}, v_{A_{B}}$ whose i-v membership and non membership function, of each element (x,y) $\square \mathrm{X} \times \mathrm{X}$ and defined by $\bar{\mu}_{A_{B}}(\mathrm{x}, \mathrm{y})=\mathrm{r} \min \left\{\bar{\mu}_{\mathrm{B}}(\mathrm{x}), \bar{\mu}_{\mathrm{B}}(\mathrm{y})\right\} \& \bar{v}_{A_{B}} \quad(\mathrm{x}, \mathrm{y})=\mathrm{r}$ $\max \left\{\bar{v}_{\mathrm{B}}(\mathrm{x}), \overline{\mathrm{v}}_{\mathrm{B}}(\mathrm{y})\right\}$

Definition 4.6Let $\mathrm{B}=\left[\left\langle\mu_{\square}^{\square}, \mu_{\square}^{\square}\right\rangle,\left\langle v_{\square}, v_{\square}\right\rangle\right]$ be an i-v subset in a set X, then the strongest i-v intuitionistic fuzzy relation on $\mathrm{X}$ that is a i-v $\mathrm{A}$ on $\mathrm{B}$ is $\mathrm{A}_{\mathrm{B}}$ and defined by, $A_{B}=\left[\left\langle\mu_{A_{B}}^{L}, \mu_{A_{B}}^{U}\right\rangle,\left\langle v_{A_{B}}^{L}, v_{A_{B}}^{U}\right\rangle\right]$

Theorem 4.7Let $\mathrm{B}=\left[\left\langle\mu_{A_{B}}^{L}, \mu_{A_{B}}^{U}\right\rangle,\left\langle v_{A_{B}}^{L}, v_{A_{B}}^{U}\right\rangle\right]$ be an i-v subset in a set $\mathrm{X}$ and $A_{B}=\left[\left\langle\mu_{A_{B}}^{L}, \mu_{A_{B}}^{U}\right\rangle,\left\langle v_{A_{B}}^{L}, v_{A_{B}}^{U}\right\rangle\right]$ be the strongest $\mathrm{i}-\mathrm{v}$ intuitionistic fuzzy relation on $\mathrm{X}$. then $\mathrm{B}$ is an $\mathrm{i}-\mathrm{v}$ intuitionistic R-ideal of $X$ if and only if $A_{B}$ is an i-v intuitionistic fuzzy R-ideal of $X \times X$.

Proof: Let $B$ be an i-v intuitionistic fuzzy a-ideal of $X$. then $\bar{\mu}_{A B}(0,0)=r \min \left\{\bar{\mu}_{\mathrm{B}}(0), \bar{\mu}_{\mathrm{B}}(0)\right\}$

$\geq \mathrm{r} \min \left\{\bar{\mu}_{\mathrm{B}}(\mathrm{x}), \bar{\mu}_{\mathrm{B}}(\mathrm{y})\right\}=\bar{\mu}_{\mathrm{AB}}(\mathrm{x}, \mathrm{y})$ and $\overline{\mathrm{v}}_{\mathrm{AB}}(0,0)=\mathrm{r} \max \left\{\overline{\mathrm{v}}_{\mathrm{B}}(0), \overline{\mathrm{v}}_{\mathrm{B}}(0)\right\} \leq \mathrm{r} \max \left\{\overline{\mathrm{v}}_{\mathrm{B}}(\mathrm{x}), \overline{\mathrm{v}}_{\mathrm{B}}(\mathrm{y})\right\}=\overline{\mathrm{v}}_{\mathrm{AB}}(\mathrm{x}, \mathrm{y}) \forall(\mathrm{x}, \mathrm{y}) \square \mathrm{X} \times \mathrm{X}$.

On the other hand $\bar{\mu}_{A_{B}}\left(\left(\mathrm{x}_{1}, \mathrm{x}_{2}\right)\right)=\bar{\mu}_{\mathrm{AB}}\left(\mathrm{x}_{1}, \mathrm{x}_{2}\right)$

$$
=\mathrm{r} \min \left\{\bar{\mu}_{\mathrm{B}}\left(\mathrm{x}_{1}\right), \bar{\mu}_{\mathrm{B}}\left(\mathrm{x}_{2}\right)\right\}
$$

$\geq \mathrm{r} \min \left\{\mathrm{r} \min \left\{\bar{\mu}_{\mathrm{B}}\left(\left(\mathrm{x}_{1} * \mathrm{z}_{1}\right) *\left(\mathrm{z}_{1} * \mathrm{y}_{1}\right)\right), \bar{\mu}_{\mathrm{B}}\left(\mathrm{y}_{1}\right)\right\}, \mathrm{r} \min \left\{\bar{\mu}_{\mathrm{B}}\left(\left(\mathrm{x}_{2} * \mathrm{z}_{2}\right) *\left(\mathrm{z}_{2} * \mathrm{y}_{2}\right)\right), \bar{\mu}_{\mathrm{B}}\left(\mathrm{y}_{2}\right)\right\}\right\}$

$=\mathrm{r} \min \left\{\mathrm{r} \min \left\{\bar{\mu}_{\mathrm{B}}\left(\left(\mathrm{x}_{1} * \mathrm{z}_{1}\right) *\left(\mathrm{z}_{1} * \mathrm{y}_{1}\right)\right), \bar{\mu}_{\mathrm{B}}\left(\left(\mathrm{x}_{2} * \mathrm{z}_{2}\right) *\left(\mathrm{z}_{2} * \mathrm{y}_{2}\right)\right)\right\}, \mathrm{r} \min \left\{\bar{\mu}_{\mathrm{B}}\left(\mathrm{y}_{1}\right), \bar{\mu}_{\mathrm{B}}\left(\mathrm{y}_{2}\right)\right\}\right\}$

$=\mathrm{r} \min \left\{\bar{\mu}_{\mathrm{AB}}\left(\left(\mathrm{x}_{1} * \mathrm{z}_{1}\right) *\left(\mathrm{z}_{1} * \mathrm{y}_{1}\right),\left(\mathrm{x}_{2} * \mathrm{z}_{2}\right) *\left(\mathrm{z}_{2} * \mathrm{y}_{2}\right)\right), \bar{\mu}_{\mathrm{AB}}\left(\mathrm{y}_{1}, \mathrm{y}_{2}\right)\right\}$

$=r \min \left\{\bar{\mu}_{\mathrm{AB}}\left(\left(\left(\mathrm{x}_{1}, \mathrm{x}_{2}\right) *\left(\mathrm{z}_{1}, \mathrm{z}_{2}\right)\right) *\left(\left(\mathrm{z}_{1}, \mathrm{z}_{2}\right) *\left(\mathrm{y}_{1}, \mathrm{y}_{2}\right)\right)\right), \bar{\mu}_{\mathrm{AB}}\left(\mathrm{y}_{1}, \mathrm{y}_{2}\right)\right\}$

Also, $\bar{v}_{A_{B}}\left(\left(\mathrm{x}_{1}, \mathrm{x}_{2}\right)\right)=\mathrm{r} \max \left\{\overline{\mathrm{v}}_{\mathrm{B}}\left(\mathrm{x}_{1}\right), \overline{\mathrm{v}}_{\mathrm{B}}\left(\mathrm{x}_{2}\right)\right\}$

$$
\leq \mathrm{r} \max \left\{\mathrm{r} \max \left\{\bar{v}_{\mathrm{B}}\left(\left(\mathrm{x}_{1} * \mathrm{z}_{1}\right) *\left(\mathrm{z}_{1} * \mathrm{y}_{1}\right)\right), \overline{\mathrm{B}}_{\mathrm{B}}\left(\mathrm{y}_{1}\right)\right\}, \mathrm{r} \max \left\{\overline{\mathrm{v}}_{\mathrm{B}}\left(\left(\mathrm{x}_{2} * \mathrm{z}_{2}\right) *\left(\mathrm{z}_{2} * \mathrm{y}_{2}\right)\right), \overline{\mathrm{v}}_{\mathrm{B}}\left(\mathrm{y}_{2}\right)\right\}\right\}
$$

$=\mathrm{r} \max \left\{\mathrm{r} \max \left\{\overline{\mathrm{v}}_{\mathrm{B}}\left(\left(\mathrm{x}_{1} * \mathrm{z}_{1}\right) *\left(\mathrm{z}_{1} * \mathrm{y}_{1}\right)\right), \overline{\mathrm{v}}_{\mathrm{B}}\left(\left(\mathrm{x}_{2} * \mathrm{z}_{2}\right) *\left(\mathrm{z}_{2} * \mathrm{y}_{2}\right)\right)\right\}, \mathrm{r} \max \left\{\overline{\mathrm{v}}_{\mathrm{B}}\left(\mathrm{z}_{1}\right), \overline{\mathrm{v}}_{\mathrm{B}}\left(\mathrm{z}_{2}\right)\right\}\right\}$

$$
\begin{aligned}
& =r \max \left\{\bar{v}_{\mathrm{AB}}\left(\left(\mathrm{x}_{1} * \mathrm{z}_{1}\right) *\left(\mathrm{z}_{1} * \mathrm{y}_{1}\right),\left(\mathrm{x}_{2} * \mathrm{z}_{2}\right) *\left(\mathrm{z}_{2} * \mathrm{y}_{2}\right)\right), \bar{v}_{\mathrm{AB}}\left(\mathrm{y}_{1}, \mathrm{y}_{2}\right)\right\} \\
& =\mathrm{r} \max \left\{\overline{\mathrm{v}}_{\mathrm{AB}}\left(\left(\left(\mathrm{x}_{1}, \mathrm{x}_{2}\right) *\left(\mathrm{z}_{1}, \mathrm{z}_{2}\right)\right) *\left(\left(\mathrm{z}_{1}, \mathrm{z}_{2}\right) *\left(\mathrm{y}_{1}, \mathrm{y}_{2}\right)\right)\right), \overline{\mathrm{v}}_{\mathrm{AB}}\left(\mathrm{z}_{1}, \mathrm{z}_{2}\right)\right\}
\end{aligned}
$$

For all $\left(\mathrm{x}_{1}, \mathrm{x}_{2}\right),\left(\mathrm{y}_{1}, \mathrm{y}_{2}\right),\left(\mathrm{z}_{1}, \mathrm{z}_{2}\right)$ in $\mathrm{X} \times \mathrm{X}$. hence $\mathrm{A}_{\mathrm{B}}$ is an $\mathrm{i}-\mathrm{v}$ intuitionistic fuzzy R-ideal of $\mathrm{X} \times \mathrm{X}$.

Conversely, let $A_{B}$ be an i-v intuitionistic fuzzy R-ideal of $X \times X$. then for all $(\mathrm{x}, \mathrm{x}) \square \mathrm{X} \times \mathrm{X}$. we have

$\mathrm{r} \min \left\{\bar{\mu}_{\mathrm{B}}(0), \bar{\mu}_{\mathrm{B}}(0)\right\}=\bar{\mu}_{\mathrm{AB}}(0,0) \geq \bar{\mu}_{\mathrm{AB}}(\mathrm{x}, \mathrm{x})=\mathrm{r} \min \left\{\bar{\mu}_{\mathrm{B}}(\mathrm{x}), \bar{\mu}_{\mathrm{B}}(\mathrm{x})\right\}($ or $) \bar{\mu}_{\mathrm{B}}(0) \geq \bar{\mu}_{\mathrm{B}}(\mathrm{x})$ and

$\mathrm{r} \max \left\{\bar{v}_{\mathrm{B}}(0), \bar{v}_{\mathrm{B}}(0)\right\}=\overline{\mathrm{v}}_{\mathrm{AB}}(0,0) \leq \bar{v}_{\mathrm{AB}}(\mathrm{x}, \mathrm{x})=\operatorname{rmin}\left\{\overline{\mathrm{v}}_{\mathrm{B}}(\mathrm{x}), \bar{\mu}_{\mathrm{B}}(\mathrm{x})\right\}($ or $) \overline{\mathrm{v}}_{\mathrm{B}}(0) \leq \overline{\mathrm{v}}_{\mathrm{B}}(\mathrm{x}) \forall \mathrm{x} \square \mathrm{X}$. Now,

let $\left(\mathrm{x}_{1}, \mathrm{x}_{2}\right),\left(\mathrm{y}_{1}, \mathrm{y}_{2}\right),\left(\mathrm{z}_{1}, \mathrm{z}_{2}\right) \square \mathrm{X} \times \mathrm{X}$, then

$\operatorname{rmin}\left\{\bar{\mu}_{\mathrm{B}}\left(\mathrm{x}_{1}, \mathrm{x}_{2}\right)\right\}=\bar{\mu}_{\mathrm{AB}}\left(\mathrm{x}_{1}, \mathrm{x}_{2}\right) \geq \mathrm{r} \min \left\{\bar{\mu}_{\mathrm{AB}}\left(\left(\left(\mathrm{x}_{1}, \mathrm{x}_{2}\right) *\left(\left(\mathrm{z}_{1}, \mathrm{z}_{2}\right)\right) *\left(\left(\mathrm{z}_{1}, \mathrm{z}_{2}\right) *\left(\mathrm{y}_{1}, \mathrm{y}_{2}\right)\right)\right), \bar{\mu}_{\mathrm{AB}}\left(\mathrm{y}_{1}, \mathrm{y}_{2}\right)\right\}\right.$

$=r \min \left\{\bar{\mu}_{\mathrm{AB}}\left(\left(\mathrm{x}_{1} * \mathrm{z}_{1}\right) *\left(\mathrm{z}_{1} * \mathrm{y}_{1}\right),\left(\mathrm{x}_{2} * \mathrm{z}_{2}\right) *\left(\mathrm{z}_{2} * \mathrm{y}_{2}\right)\right), \bar{\mu}_{\mathrm{AB}}\left(\mathrm{y}_{1}, \mathrm{y}_{2}\right)\right\}$

$=\mathrm{r} \min \left\{\mathrm{r} \min \left\{\bar{\mu}_{\mathrm{B}}\left(\left(\mathrm{x}_{1} * \mathrm{z}_{1}\right) *\left(\mathrm{z}_{1} * \mathrm{y}_{1}\right)\right), \bar{\mu}_{\mathrm{B}}\left(\mathrm{z}_{1}\right)\right\}, \mathrm{r} \min \left\{\bar{\mu}_{\mathrm{AB}}\left(\left(\mathrm{x}_{2} * \mathrm{z}_{2}\right) *\left(0 * \mathrm{y}_{2}\right)\right), \bar{\mu}_{\mathrm{B}}\left(\mathrm{z}_{2}\right)\right\}\right\}$

Also, $\operatorname{rmax}\left\{\overline{\mathrm{v}}_{\mathrm{B}}\left(\mathrm{x}_{1}, \mathrm{x}_{2}\right)\right\}=\overline{\mathrm{v}}_{\mathrm{AB}}\left(\mathrm{x}_{1}, \mathrm{x}_{2}\right)$

$\leq \mathrm{r} \max \left\{\overline{\mathrm{v}}_{\mathrm{AB}}\left(\left(\left(\mathrm{x}_{1}, \mathrm{x}_{2}\right) *\left(\left(\mathrm{z}_{1}, \mathrm{z}_{2}\right)\right) *\left(\left(\mathrm{z}_{1}, \mathrm{z}_{2}\right) *\left(\mathrm{y}_{1}, \mathrm{y}_{2}\right)\right)\right), \overline{\mathrm{v}}_{\mathrm{AB}}\left(\mathrm{y}_{1}, \mathrm{y}_{2}\right)\right\}\right.$

$=\mathrm{r} \max \left\{\overline{\mathrm{v}}_{\mathrm{AB}}\left(\left(\mathrm{x}_{1} * \mathrm{z}_{1}\right) *\left(\mathrm{z}_{1} * \mathrm{y}_{1}\right),\left(\mathrm{x}_{2} * \mathrm{z}_{2}\right) *\left(\mathrm{z}_{2} * \mathrm{y}_{2}\right)\right), \overline{\mathrm{v}}_{\mathrm{AB}}\left(\mathrm{y}_{1}, \mathrm{y}_{2}\right)\right\}$

$=\mathrm{r} \max \left\{\mathrm{r} \max \left\{\overline{\mathrm{v}}_{\mathrm{B}}\left(\left(\mathrm{x}_{1} * \mathrm{z}_{1}\right) *\left(\mathrm{z}_{1} * \mathrm{y}_{1}\right)\right), \bar{\mu}_{\mathrm{B}}\left(\mathrm{y}_{1}\right)\right\}, r \max \left\{\overline{\mathrm{v}}_{\mathrm{AB}}\left(\left(\mathrm{x}_{2} * \mathrm{z}_{2}\right) *\left(\mathrm{z}_{2} * \mathrm{y}_{2}\right)\right), \overline{\mathrm{v}}_{\mathrm{B}}\left(\mathrm{y}_{2}\right)\right\}\right\}$

If $\mathrm{x}_{2}=\mathrm{y}_{2}=\mathrm{z}_{2}=0$, then $\mathrm{r} \min \left\{\bar{\mu}_{\mathrm{B}}\left(\mathrm{x}_{1}\right), \bar{\mu}_{\mathrm{B}}(0)\right\} \geq \mathrm{r} \min \left\{\mathrm{r} \min \left\{\bar{\mu}_{\mathrm{B}}\left(\left(\mathrm{x}_{1} * \mathrm{z}_{1}\right) *\left(\mathrm{z}_{1} * \mathrm{y}_{1}\right), \bar{\mu}_{\mathrm{B}}\left(\mathrm{y}_{1}\right)\right\}, \bar{\mu}_{\mathrm{B}}(0)\right\}\right.$ and

$\mathrm{r} \max \left\{\overline{\mathrm{v}}_{\mathrm{B}}\left(\mathrm{x}_{1}\right), \overline{\mathrm{v}}_{\mathrm{B}}(0)\right\} \geq \mathrm{r} \max \left\{\mathrm{r} \max \left\{\overline{\mathrm{v}}_{\mathrm{B}}\left(\left(\mathrm{x}_{1} * \mathrm{z}_{1}\right) *\left(\mathrm{z}_{1} * \mathrm{y}_{1}\right), \overline{\mathrm{v}}_{\mathrm{B}}\left(\mathrm{y}_{1}\right)\right\}, \overline{\mathrm{v}}_{\mathrm{B}}(0)\right\}\right.$

$\bar{\mu}_{\mathrm{B}}\left(\mathrm{x}_{1}\right) \geq \mathrm{r} \min \left\{\bar{\mu}_{\mathrm{B}}\left(\left(\mathrm{x}_{1}{ }^{*} \mathrm{z}_{1}\right) *\left(\mathrm{z}_{1}{ }^{*} \mathrm{y}_{1}\right), \bar{\mu}_{\mathrm{B}}\left(\mathrm{y}_{1}\right)\right\}\right.$ and

$\bar{v}_{\mathrm{B}}\left(\mathrm{x}_{1}\right) \geq \mathrm{r} \max \left\{\overline{\mathrm{v}}_{\mathrm{B}}\left(\left(\mathrm{x}_{1} * \mathrm{z}_{1}\right) *\left(\mathrm{z}_{1} * \mathrm{y}_{1}\right), \overline{\mathrm{v}}_{\mathrm{B}}\left(\mathrm{y}_{1}\right)\right\}\right.$.

Therefore $\mathrm{B}$ is $\mathrm{i}-\mathrm{v}$ intuitionistic fuzzy $\mathrm{R}$-ideal of $\mathrm{X}$.

Theorem 4.8: If $\bar{\mu}_{\mathrm{A}}$ is a i-v intuitionistic fuzzy a-ideal of BCI-algebra X, then $\bar{\mu}_{A^{m}}$ is also i-v intuitionistic fuzzy

R-ideal of BCI-algebra X

Proof: For all $\mathrm{x}, \mathrm{y}, \mathrm{z} \in \mathrm{X}$

$$
\begin{aligned}
& \text { 1. } \bar{\mu}_{A}(0) \geq \bar{\mu}_{A}(x), \bar{v}_{A}(0) \leq \bar{v}_{A}(x) . \quad\left[\bar{\mu}_{A}(0)\right]^{m} \geq\left[\bar{\mu}_{A}(x)\right],\left[\bar{v}_{A}(0)\right]^{m} \leq\left[\bar{v}_{A}(x)\right] \\
& \bar{\mu}_{A}(0)^{m} \geq \bar{\mu}_{A}(x)^{m}, \bar{v}_{A}(0)^{m} \leq \bar{v}_{A}(x)^{m} . \quad \bar{\mu}_{A^{m}}(0) \geq \bar{\mu}_{A^{m}}(x), \bar{v}_{A^{m}}(0) \leq \bar{v}_{A^{m}}(x) \quad \forall x \in X
\end{aligned}
$$


2. $\bar{\mu}_{A}(x) \geq r \min \left\{\bar{\mu}_{A}\left((x * z) *\left(\mathrm{z}^{*} y\right)\right), \bar{\mu}_{A}(\mathrm{y})\right\} \cdot\left[\bar{\mu}_{A}(x)\right]^{m} \geq\left[r \min \left\{\bar{\mu}_{A}\left((x * z) *\left(\mathrm{z}^{*} y\right)\right), \bar{\mu}_{A}(\mathrm{y})\right\}\right]^{m}$ $\bar{\mu}_{A}(x)^{m} \geq r \min \left\{\bar{\mu}_{A}\left((x * z) *\left(\mathrm{z}^{*} y\right)\right), \bar{\mu}_{A}(\mathrm{y})\right\}^{m} \cdot \bar{\mu}_{A^{m}}(x) \geq r \min \left\{\bar{\mu}_{A}\left(\left(x^{*} z\right)^{*}\left(\mathrm{z}^{*} y\right)\right)^{m}, \bar{\mu}_{A}(\mathrm{y})^{m}\right\}$ $\bar{\mu}_{A^{m}}(x) \geq r \min \left\{\bar{\mu}_{A^{m}}\left((x * z) *\left(\mathrm{z}^{*} y\right)\right) \bar{\mu}_{A^{m}}(\mathrm{y})\right\}$

3. $\bar{v}_{A}(x) \leq r \max \left\{\bar{\mu}_{A}\left(\left(x^{*} z\right)^{*}\left(\mathrm{z}^{*} y\right)\right), \bar{\mu}_{A}(\mathrm{y})\right\} \cdot\left[\bar{v}_{A}(x)\right]^{m} \leq\left[r \max \left\{\bar{v}_{A}\left((x * z) *\left(\mathrm{z}^{*} y\right)\right) \bar{v}_{A}(\mathrm{y})\right\}\right]^{m}$ $\bar{v}_{A}(x)^{m} \leq r \max \left\{\bar{v}_{A}\left((x * z) *\left(\mathrm{z}^{*} y\right)\right), \bar{v}_{A}(\mathrm{y})\right\}^{m} \cdot \bar{v}_{A^{m}}(x) \leq r \max \left\{\bar{v}_{A}\left(\left(x^{*} z\right)^{*}\left(\mathrm{z}^{*} y\right)\right)^{m}, \bar{v}_{A}(\mathrm{y})^{m}\right\}$ $\bar{v}_{A^{m}}(x) \leq r \max \left\{\bar{v}_{A^{m}}\left((x * z) *\left(\mathrm{z}^{*} y\right)\right) \bar{v}_{A^{m}}(\mathrm{y})\right\}$

Theorem 4.9:If $\bar{\mu}_{\mathrm{A}}$ is a i-v intuitionistic fuzzy R-ideal of BCI-algebra X, then $\bar{\mu}_{A \cap B}$ isalso a i-v intuitionistic fuzzy R-ideal of BCI-algebra X

Proof: For all $\mathrm{x}, \mathrm{y}, \mathrm{z} \in \mathrm{X}$

1. $\bar{\mu}_{A}(0) \geq \bar{\mu}_{A}(x), \bar{v}_{A}(0) \leq \bar{v}_{A}(x)$ and $\bar{\mu}_{B}(0) \geq \bar{\mu}_{B}(x), \bar{v}_{B}(0) \leq \bar{v}_{B}(x)$

$\min \left\{\bar{\mu}_{A}(0), \bar{\mu}_{B}(0)\right\} \geq \min \left\{\bar{\mu}_{A}(x), \bar{\mu}_{B}(x)\right\}, \min \left\{\bar{v}_{A}(0), \bar{v}_{B}(0)\right\} \leq \min \left\{\bar{v}_{A}(x), \bar{v}_{B}(x)\right\}$

$\bar{\mu}_{A \cap B}(0) \geq \bar{\mu}_{A \cap B}(x), \bar{v}_{A \cap B}(0) \leq \bar{v}_{A \cap B}(x)$

2. $\bar{\mu}_{A}(x) \geq r \min \left\{\bar{\mu}_{A}\left(\left(x^{*} z\right)^{*}\left(\mathrm{z}^{*} y\right)\right), \bar{\mu}_{A}(\mathrm{y})\right\}, \bar{\mu}_{B}\left(y^{*} x\right) \geq r \min \left\{\bar{\mu}_{B}\left(\left(x^{*} z\right)^{*}\left(\mathrm{z}^{*} y\right)\right), \bar{\mu}_{A}(\mathrm{y})\right\}$

$\left\{\bar{\mu}_{A}(x), \bar{\mu}_{B}(x)\right\} \geq\left\{r \min \left\{\bar{\mu}_{A}\left(\left(x^{*} z\right)^{*}\left(\mathrm{z}^{*} y\right)\right), \bar{\mu}_{A}(\mathrm{y})\right\}, r \min \left\{\bar{\mu}_{B}\left(\left(x^{*} z\right)^{*}\left(\mathrm{z}^{*} y\right)\right), \bar{\mu}_{B}(\mathrm{y})\right\}\right\}$

$\min \left\{\bar{\mu}_{A}(x), \bar{\mu}_{B}(x)\right\} \geq \min \left\{r \min \left\{\bar{\mu}_{A}\left((x * z) *\left(\mathrm{z}^{*} y\right)\right), \bar{\mu}_{A}(\mathrm{y})\right\}, r \min \left\{\bar{\mu}_{B}\left(\left(x^{*} z\right)^{*}\left(\mathrm{z}^{*} y\right)\right), \bar{\mu}_{B}(\mathrm{y})\right\}\right\}$

$\geq \min \left\{r \min \left\{\bar{\mu}_{A}\left(\left(x^{*} z\right)^{*}\left(\mathrm{z}^{*} y\right)\right), \bar{\mu}_{B}\left(\left(x^{*} z\right)^{*}\left(\mathrm{z}^{*} y\right)\right)\right\}, r \min \left\{\bar{\mu}_{A}(\mathrm{y}), \bar{\mu}_{B}(\mathrm{y})\right\}\right\}$

$\bar{\mu}_{A \cap B}(x) \geq r \min \left\{\bar{\mu}_{A \cap B}\left(\left(x^{*} z\right) *\left(\mathrm{z}^{*} y\right)\right), \bar{\mu}_{A \cap B}(\mathrm{y})\right\}$

3. $\bar{v}_{A}(x) \leq r \max \left\{\bar{v}_{A}\left((x * z) *\left(\mathrm{z}^{*} y\right)\right), \bar{v}_{A}(\mathrm{y})\right\}, \bar{v}_{B}(x) \leq r \max \left\{\bar{v}_{B}\left(\left(x^{*} z\right) *\left(\mathrm{z}^{*} y\right)\right), \bar{v}_{A}(\mathrm{y})\right\}$

$\left\{\bar{v}_{A}(x), \bar{v}_{B}(x)\right\} \leq\left\{r \max \left\{\bar{v}_{A}\left(\left(x^{*} z\right) *\left(\mathrm{z}^{*} y\right)\right), \bar{v}_{A}(\mathrm{y})\right\}, r \max \left\{\bar{v}_{B}\left(\left(x^{*} z\right)^{*}\left(\mathrm{z}^{*} y\right)\right), \bar{v}_{B}(\mathrm{y})\right\}\right\}$

If one is contained in the other

$\min \left\{\bar{v}_{A}(x), \bar{v}_{B}(x)\right\} \leq \min \left\{r \max \left\{\bar{v}_{A}\left((x * z) *\left(\mathrm{z}^{*} y\right)\right), \bar{v}_{A}(z)\right\}, r \max \left\{\bar{v}_{B}\left(\left(x^{*} z\right) *\left(\mathrm{z}^{*} y\right)\right), \bar{v}_{B}(\mathrm{y})\right\}\right\}$

$\bar{v}_{A \cap B}(x) \leq r \max \left\{\min \left\{\bar{v}_{A}\left((x * z) *\left(\mathrm{z}^{*} y\right)\right), \bar{v}_{B}\left(\left(x^{*} z\right) *\left(\mathrm{z}^{*} y\right)\right)\right\}, \min \left\{\bar{v}_{A}(z), \bar{v}_{B}(\mathrm{y})\right\}\right\}$

$\bar{v}_{A \cap B}(x) \leq r \max \left\{\bar{v}_{A \cap B}\left((x * z) *\left(z^{*} y\right)\right), \bar{v}_{A \cap B}(\mathrm{y})\right\}$

Theorem 4.10: If $\bar{\mu}_{\mathrm{A}}$ is a i-v intuitionistic fuzzy R-ideal of BCI-algebra X, then $\bar{\mu}_{A \cup B}$ isalso a i-v intuitionistic fuzzy R-ideal of BCI-algebra X.

Proof: For all $\mathrm{x}, \mathrm{y}, \mathrm{z} \in \mathrm{X}$

1. $\bar{\mu}_{A}(0) \geq \bar{\mu}_{A}(x), \bar{v}_{A}(0) \leq \bar{v}_{A}(x)$ and $\bar{\mu}_{B}(0) \geq \bar{\mu}_{B}(x), \bar{v}_{B}(0) \leq \bar{v}_{B}(x)$

$\min \left\{\bar{\mu}_{A}(0), \bar{\mu}_{B}(0)\right\} \geq \min \left\{\bar{\mu}_{A}(x), \bar{\mu}_{B}(x)\right\}, \min \left\{\bar{v}_{A}(0), \bar{v}_{B}(0)\right\} \leq \min \left\{\bar{v}_{A}(x), \bar{v}_{B}(x)\right\}$

$\bar{\mu}_{A \cup B}(0) \geq \bar{\mu}_{A \cup B}(x), \bar{v}_{A \cup B}(0) \leq \bar{v}_{A \cup B}(x)$

2. $\bar{\mu}_{A}(x) \geq r \min \left\{\bar{\mu}_{A}\left(\left(x^{*} z\right)^{*}\left(\mathrm{z}^{*} y\right)\right), \bar{\mu}_{A}(\mathrm{y})\right\}, \quad \bar{\mu}_{B}(x) \geq r \min \left\{\bar{\mu}_{B}\left(\left(x^{*} z\right)^{*}\left(\mathrm{z}^{*} y\right)\right), \bar{\mu}_{A}(\mathrm{y})\right\}$

$\left\{\bar{\mu}_{A}(x), \bar{\mu}_{B}(x)\right\} \geq\left\{r \min \left\{\bar{\mu}_{A}\left((x * z) *\left(\mathrm{z}^{*} y\right)\right), \bar{\mu}_{A}(\mathrm{y})\right\}, r \min \left\{\bar{\mu}_{B}\left(\left(x^{*} z\right)^{*}\left(\mathrm{z}^{*} y\right)\right), \bar{\mu}_{B}(\mathrm{y})\right\}\right\}$

$\max \left\{\bar{\mu}_{A}(x), \bar{\mu}_{B}(x)\right\} \geq \max \left\{r \min \left\{\bar{\mu}_{A}\left(\left(x^{*} z\right)^{*}\left(\mathrm{z}^{*} y\right)\right), \bar{\mu}_{A}(\mathrm{y})\right\}, r \min \left\{\bar{\mu}_{B}\left(\left(x^{*} z\right)^{*}\left(\mathrm{z}^{*} y\right)\right), \bar{\mu}_{B}(\mathrm{y})\right\}\right\}$

$\geq \max \left\{r \min \left\{\bar{\mu}_{A}\left(\left(x^{*} z\right)^{*}\left(\mathrm{z}^{*} y\right)\right), \bar{\mu}_{B}\left(\left(x^{*} z\right)^{*}\left(\mathrm{z}^{*} y\right)\right)\right\}, \quad r \max \left\{\bar{\mu}_{A}(\mathrm{y}), \bar{\mu}_{B}(\mathrm{y})\right\}\right\}$

If one is contained in the other

$\mathrm{r} \min \left\{\max \left\{\bar{\mu}_{A}\left(\left(x^{*} z\right)^{*}\left(\mathrm{z}^{*} y\right)\right), \bar{\mu}_{B}\left(\left(x^{*} z\right)^{*}\left(\mathrm{z}^{*} y\right)\right)\right\}, \quad \max \left\{\bar{\mu}_{A}(\mathrm{y}), \bar{\mu}_{B}(\mathrm{y})\right\}\right\}$

$\bar{\mu}_{A \cup B}(x) \geq r \min \left\{\bar{\mu}_{A \cup B}\left(\left(x^{*} z\right)^{*}\left(\mathrm{z}^{*} y\right)\right), \bar{\mu}_{A \cup B}(\mathrm{y})\right\}$

3. $\bar{v}_{A}(x) \leq r \max \left\{\bar{v}_{A}\left((x * z) *\left(\mathrm{z}^{*} y\right)\right), \bar{v}_{A}(\mathrm{y})\right\}, \quad \bar{v}_{B}(x) \leq r \max \left\{\bar{v}_{B}\left((x * z) *\left(\mathrm{z}^{*} y\right)\right), \bar{\mu}_{A}(\mathrm{y})\right\}$

$\left\{\bar{v}_{A}(x), \bar{v}_{B}(x)\right\} \leq\left\{r \max \left\{\bar{v}_{A}\left((x * z) *\left(\mathrm{z}^{*} y\right)\right), \bar{v}_{A}(\mathrm{y})\right\}, r \max \left\{\bar{v}_{B}\left(\left(x^{*} z\right) *\left(\mathrm{z}^{*} y\right)\right), \bar{v}_{B}(z)\right\}\right\}$

$\max \left\{\bar{v}_{A}(x), \bar{v}_{B}(x)\right\} \leq \max \left\{r \max \left\{\bar{v}_{A}\left(\left(x^{*} z\right)^{*}\left(\mathrm{z}^{*} y\right)\right), \bar{v}_{A}(\mathrm{y})\right\}, r \max \left\{\bar{v}_{B}\left((x * z) *\left(\mathrm{z}^{*} y\right)\right), \bar{v}_{B}(\mathrm{y})\right\}\right\}$

$\bar{v}_{A \cup B}(x) \leq r \max \left\{\max \left\{\bar{v}_{A}\left(\left(x^{*} z\right)^{*}\left(\mathrm{z}^{*} y\right)\right), \bar{v}_{B}\left(\left(x^{*} z\right)^{*}\left(\mathrm{z}^{*} y\right)\right)\right\}, \max \left\{\bar{v}_{A}(\mathrm{y}), \bar{v}_{B}(\mathrm{y})\right\}\right\}$

$\bar{v}_{A \cup B}(x) \leq r \max \left\{\bar{v}_{A \cup B}\left(\left(x^{*} z\right)^{*}\left(\mathrm{z}^{*} y\right)\right), \bar{v}_{A \cup B}(\mathrm{y})\right\}$ 


\section{References:}

[1]. K.T Atanassov, intuitionisticfuzzy sets and systems, 20(1986), 87-96

[2]. K.T Atanassov, intuitionisticfuzzy sets. Theory and applications, studies in fuzziness and soft computing, 35.Heidelberg; physicaverlag

[3]. R.Biswas, Rosenfeld's fuzzy subgroups with interval-valued membership functions, fuzzy sets and systems 63(1994), no.1,87-90

[4]. S.M. Hong, Y.B.Kim and G.I.Kim, fuzzy BCI-sub algebras with interval-valued membership functions, math japonica, 40(2)(1993)199-202

[5]. K.Iseki, an algebra related with a propositional calculus, proc, Japan Acad.42 (1966),26-29

[6]. H.M.Khalid, B.Ahmad, fuzzy H-ideals in BCI-algebras, fuzzy sets and systems 101(1999)153-158.

[7]. L.A.zadeh, the concept of a linguistic variable and its application to approximate reasoning. I, information sci,8(1975),199-249. 